\title{
A new approach to face recognition based on features fusion
}

\author{
Yanhong $\mathrm{Fu}$ \\ Tianjin Key Laboratory for Control Theory \& Applications in Complicated Systems \\ Tianjin University of Technology \\ 391, Binshui Xidao, Xiqing District, Tianjin, 300384, Chinafuyanhong1990@126.com
}

Keywords: pattern recognition; two dimensional principal component (2DPCA); extended LBP

\begin{abstract}
In this paper, a theoretically efficient method is developed for face recognition. It is based on two dimensional principal component (2DPCA) analysis and extended local binary pattern (Extended LBP, ELBP) texture. First, the ELBP operator is employed to extract the local texture of the face images. Second, 2DPCA is used to reduce the dimensionality of the extracted feature and get the optimal projection space. Finally, the nearest distance classification is used to distinguish each testing image. The method has been ac-cessed on ATR-Jaffe and AR face databases. Results demonstrate that the proposed method is obviously superior to PCA and 2DPCA, and its recognition rate is more stable than PCA. Meanwhile, the proposed method has strong robustness against illumination and facial expression changes.
\end{abstract}

\section{INTRODUCTION}

In the field of biometrics recognition, face recognition has good application prospects in the field of identification, security monitoring and human-computer interaction. Factors affecting the face recognition mainly lie in: the changeable facial expressions and pose [11]; the same face will change with age; two dimensional face images are susceptible to be affected by illumination when they are filmed; recognizing a specific face involves a lot of knowledge, such as image preprocessing, pattern recognition and computer vision. Feature extraction is key to face recognition. Global feature extraction methods include principal component analysis (PCA) [9], independent component analysis (ICA) [6] and linear discriminate analysis (LDA) [12] under the conditions that samples obey the multivariate normal distribution. The advantages of PCA method is to get global features which can represent the characteristics of the face image by K-L transform, the disadvantage is that two-dimensional image matrix need to be transformed into one-dimensional vector, resulting in a huge amount of computation. Meanwhile PCA has a bad robustness under the illumination, pose and facial expressions. Jian Yang developed a novel two-dimensional PCA (2DPCA) [10] method. Compared with global features, local feature has a strong robustness against the changes of illumination and pose. The common local feature extraction methods include local binary pattern (LBP) $[2,8]$, scale invariant feature transform (SIFT) and so on. LBP is an effective texture extraction method and has been extensively exploited in many applications, for instance, image analysis, texture classification [1, 5, 7], environment modeling and other fields [3]. Classifiers commonly used in face recognition are: minimum distance method, nearest neighbor classifier, support vector machine classifier and BP neural network method.

Based on 2DPCA, this paper proposed a fusion feature extraction method of 2DPCA and extended LBP (ELBP) texture, in the stage of classification a reliable algorithm - the nearest classifier - is utilized to identify a specific face image, these measures effectively reduce the influence of illumination, facial expressions and pose changes and improve the recognition rate.

\section{FUSION FEATURE EXTRACTION OF 2DPCA AND EXTENDED LBP}

The proposed method

This paper aimed to improve face recognition rate under lighting changes and facial expressions. 
Figure 1 illustrates the flow chart of the improved method.

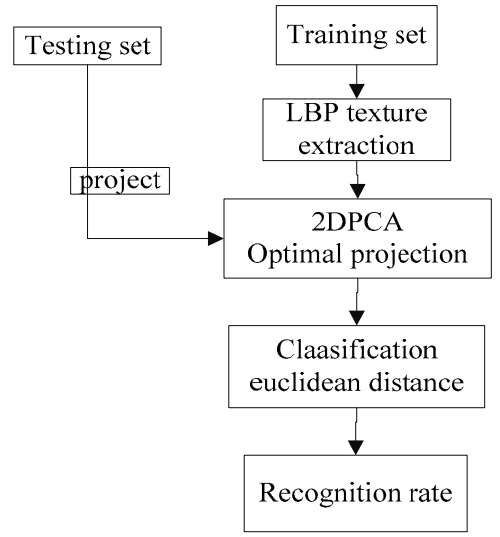

Figure 1. The structure diagram of face recognition of the proposed method

First, preprocess images to enhance information. Second, use LBP to describe faces. Third, obtain the optimal projection space by 2DPCA. Fourthly, project test set and training set into the optimal projection space and obtain a feature matrix for each image. Finally, employ a classifier to classify, summarize the recognition rate and do some analysis comparing with other similar algorithms.

\section{LBP face appearance descriptor}

LBP-based feature extraction operator is computationally efficient statistical characteristics that can distinguish different objects in the same image in microcosm form [6], and is not sensitive to changes of gray-scale and different lighting conditions. LBP operator represents texture by comparing the gray value of the center pixel with the gray-scale of its neighboring pixels. It has been proved a powerful approach to describe local structure [5].

The LBP pattern of each pixel is defined as:

$$
L B P_{P, R}=\sum_{p=0}^{P-1} s\left(I_{p}-I_{C}\right) 2^{p}
$$

where $I_{C}$ is the gray-scale of center pixel, $\mathrm{P}$ stands for the number of pixels in this neighborhood, $\mathrm{R}$ represents the radius of the neighbor, $I_{p}$ is the gray-scale of a neighbor pixel. ${ }^{s(.)}$ is defined by:

$$
s(.)=\left\{\begin{array}{l}
1, I_{p}-I_{C} \geq 0 \\
0, I_{p}-I_{C}<0
\end{array}\right.
$$

Obviously, it has strong robustness for any monotonic transformation of the gray scale. When the image is rotating, the position of pixels will also rotate a certain angle of rotation around the center. To remove the effects of rotation, Ojala defined a unique identifier :

$$
L B P_{P, R}^{r i}=\min \left\{\operatorname{ROR}\left(\operatorname{LBP} P_{P, R}, i\right) \mid i=0,1, \ldots, P-1\right\}
$$

Figure 2 illustrates how to get the rotation invariance LBP value.

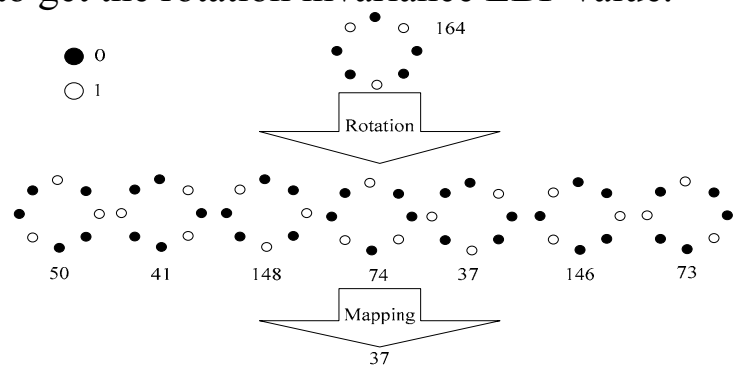

Figure 2. The uniform rotation invariant LBP. The ${ }^{L B P_{\mathrm{P}, \mathrm{R}}}$ value is $(00100101)_{2}=37$ 
Ojala proposed uniform patterns [8], i.e., after caculating, the corresponding binary sequence contains twice spatial transitions at most ( $0 / 1$ changes). Ojala believes that uniform LBP patterns providing the vast majority, sometimes over 90 percent [8]. The definition of uniform patterns is as follows :

$$
\begin{aligned}
U\left(L B P_{P, R}\right)= & \left|s\left(I_{p-1}-I_{C}\right)-s\left(I_{0}-I_{C}\right)\right| \\
& +\sum_{p=1}^{P-1} s\left(I_{p}-I_{C}\right)-s\left(I_{p-1}-I_{C}\right)
\end{aligned}
$$

The uniform rotation invariant LBP patterns (ELBP) is defined as:

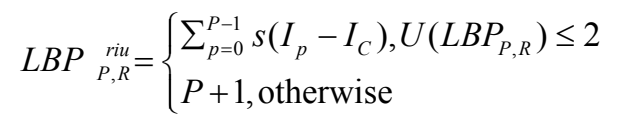

\section{DPCA feature extraction}

Turk and Pentland first used PCA feature extraction method in face recognition [9]. The basic idea of PCA is K-L transform: extract the principal component of high-dimensional image matrix.

Eigenfaces are calculated as the following steps:

a. reading samples image matrix:

$X=\left[x_{11}, x_{12}, \ldots, x_{i j}\right]^{T}, i \in 1,2, \ldots, \mathrm{M}, j \in 1,2, \ldots, \mathrm{N}$

where ${ }^{x_{i j}}$ represents the ${ }^{j}$ th picture of the ${ }^{i}$ th class, $\mathrm{M}$ classes and $\mathrm{N}$ images per subject.

b. calculate the average facial vector:

$\psi=\frac{1}{\mathrm{MN}} \sum_{i=1}^{\mathrm{M}} \sum_{j=1}^{\mathrm{N}} x_{i j}$

c. calculate the mean face:

$\varphi_{i j}=x_{i j}-\psi$

d. construct covariance matrix:

$C=\frac{1}{\mathrm{MN}} \sum_{i=1}^{\mathrm{M}} \sum_{j=1}^{N} \varphi_{i j} \varphi_{i j}{ }^{T}$

e. solve the eigenvalues and eigenvectors of the covariance matrix and constructing eigenfaces space

According to the eigenvalues's usefulness in characterizing variation, we choose $k$ eigenvalues. Then, $u_{k}$ is the relevant eigenvectors of selected eigenvalues, and eigenfaces space is: $\omega=\left[u_{1}, u_{2}, \ldots, u_{k}\right]$.

The transformed space is: $y=\omega^{T} x$ and the dimension of the original space reduced to $k$.

Obviously, 2D image matrix needs to be transformed into 1D vector in PCA. And 2DPCA calculates non-directly the eigenvectors of image scatter matrix, whose basic idea is that the size of $H=L \times R$ image matrix is projected on $X$ by a linear transition- $Y=H X$, where $Y$ is called projection feature eigenvectors of $H$.

The average image matrix of training set is:

$S=\frac{1}{M} \sum_{i=1}^{N} \sum_{j=1}^{K} S_{j}^{i}$

$S^{i}$ represents the ${ }^{j}$ th sample of the $i$ th class, $N$ classes and $K$ pictures of each class, $M=N K$. Calculate the covariance matrix of training set: 


$$
G=\frac{1}{M} \sum_{i=1}^{N} \sum_{j=1}^{K}\left(S_{j}^{i}-S\right)^{T}\left(S_{j}^{i}-S\right)
$$

Calculate the eigenvalues and eigenvectors of ${ }^{G}$, select $p(\mathrm{p}<<\mathrm{M})$ lager eigenvalues and calculate the corresponding orthogonal eigenvectors $P_{1}, P_{2}, \cdots, P_{p}$ as optimal projection. The training set $\left\{S_{j}^{i} \in R^{m \times n}, i=1,2, \cdots, N, j=1,2, \cdots, K\right\}$ is projected on the optimal projection space to get

$$
\begin{aligned}
Y_{j}^{i} & =\left[S_{j}^{i} P_{1}, S_{j}^{i} P_{2}, \cdots, S_{j}^{i} P_{p}\right] \\
& =\left[Y_{j}^{i}(1), Y_{j}^{i}(2), \cdots, Y_{j}^{i}(p)\right] \in R^{m \times p}
\end{aligned}
$$

where $Y_{j}^{i}$ is the eigenmatrix of ${ }^{S_{j}^{i}}$ and $Y_{j}^{i}(1), Y_{j}^{i}(2), \cdots, Y_{j}^{i}(p)$ is the principal component of ${ }^{S_{j}^{i}}$.

Test set is projected on the optimal projection space, i.e., $X_{i}=B P_{i}$ where $B$ is the test set matrix and $X=\{X(1), X(2), \cdots, X(p)\}$ is the projection space of test set. Finally, utilize nearest distance classifier to recognize which is defined by

$$
\begin{aligned}
& d\left(Y_{j}^{i}, X_{i}\right)=\sum_{k=1}^{p}\left\|Y_{j}^{i}(k)-X(k)\right\|^{2} \\
& \text { where }\left\|Y_{j}^{i}(k)-X(k)\right\|^{2} \text { describes the Euclidean distance between } Y_{j}^{i} \text { and } X_{i} \text {. Given a test sample B, } \\
& \text { if } d\left(\mathrm{~B}, \mathrm{~S}_{l}\right)=\min d\left(\mathrm{~B}, \mathrm{~S}_{i}^{j}\right) \text { and } \mathrm{S}_{l} \in \omega_{R} \text {, then } B \in \omega_{R} .
\end{aligned}
$$

\section{EXPERIMENTAL RESULTS}

ATR-Jaffe and AR standard face databases are used to test the performance. ATR-Jaffe database, which is different from other common male face databases, contains 10 female subjects and each subject includes 20 different images and is set up to access the performance of the method under conditions where the facial expressions are varied. The advantages of AR are its different illumination changes and occlusions (sunglasses or no sunglasses, scarves or no scarves), including 2,600 images from 50 male subjects and 50 female subjects. AR database is selected to evaluate the performance of the method under larger variations in illumination changes and occlusions. Fig. 3 shows part of these two databases.

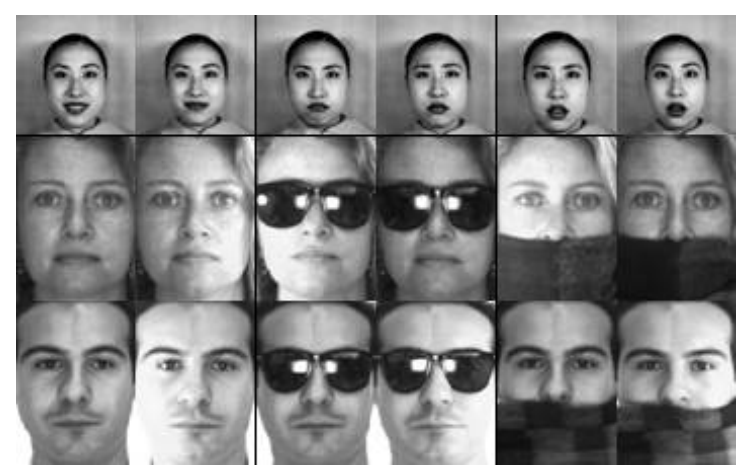

Figure 3. Some samples from the two databases

\section{Experiments on the ATR-Jaffe database}

Table 1 shows the design on ATR-Jaffe. The proposed algorithm was tested on $2.59 \mathrm{GHz} \mathrm{PC}$ and MATLAB R2011a. Before simulating experiments, the training set and the test set were preprocessed to enhance image information and all images were grayscale and normalized. Furthermore, the face region detection is necessary since there is some useless information around the area of face, for instance, hair, background, ears. Figure 4 shows the part of results after detection. Figure 5 is the experimental results on ATR-Jaffe 


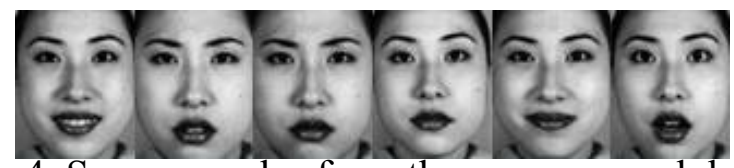

Figure 4. Some samples from the preprocessed database

Table 1. Experimental design on ATR-Jaffe

\begin{tabular}{lllllll}
\hline NO. & 1 & 2 & 3 & 4 & 5 & 6 \\
\hline Train set & $10 \times 10$ & $12 \times 10$ & $14 \times 10$ & $16 \times 10$ & $18 \times 10$ & $19 \times 10$ \\
Test set & $10 \times 10$ & $8 \times 10$ & $6 \times 10$ & $4 \times 10$ & $2 \times 10$ & $1 \times 10$ \\
\hline
\end{tabular}

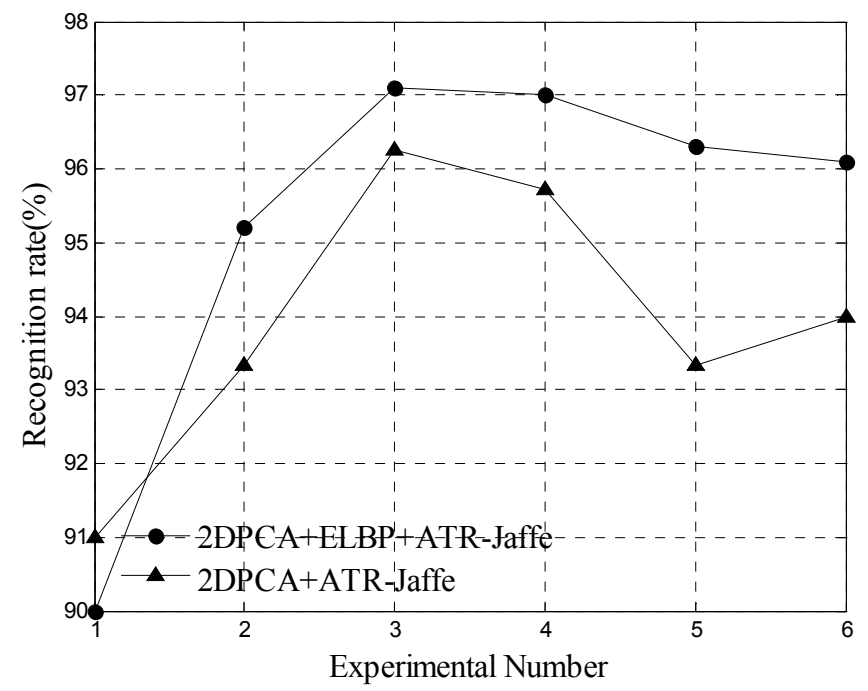

Figure 5. The experimental results on ATR-Jaffe

Table 2. The average recognition rate of several methods on ATR-Jaffe

\begin{tabular}{lllll}
\hline Methods & PCA & LBP & 2DPCA & $\begin{array}{l}\text { 2DPCA } \\
\text { +ELBP }\end{array}$ \\
\hline Average rec-rate & $0.856[4]$ & $0.810[1]$ & 0.940 & 0.953 \\
\hline
\end{tabular}

It can be seen from Table 2. and Figure 5. that the average recognition accuracy rate of the proposed method is higher and the recognition time is faster. With the reduction of the number of training samples, all this methods of recognition rate declined, but the 2DPCA + ELBP method is superior to the other algorithms.

\section{Experiments on the AR database}

The experiments was performed as the design of Table 4. Before simulating, the training set and the test set are preprocessed to enhance image information.

Table 3. The recognition rate and time on AR

\begin{tabular}{lllll}
\hline NO & 1 & 2 & 3 & 4 \\
\hline Train set & $13 \times 100$ & $17 \times 100$ & $21 \times 100$ & $25 \times 100$ \\
Test set & $13 \times 100$ & $9 \times 100$ & $5 \times 100$ & $1 \times 100$ \\
\hline
\end{tabular}




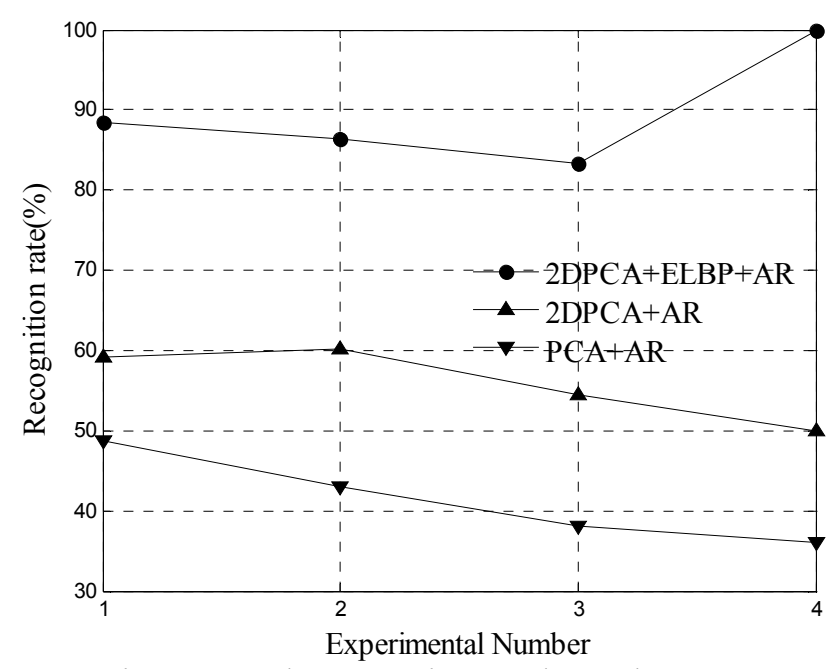

Figure 6. The experimental results on AR

From Figure 6, when the condition of training samples is complex (illumination changes and occlusions), it is apparent that the performance of the proposed method precedes the conventional recognition method. First, the recognition time of 2DPCA+ELBP is less since it omitted a step of 2D matrix to $1 \mathrm{D}$ vector. Second, the two features are a pair of complementary features.

\section{CONCLUSIONS}

How to weaken the effects of illumination changes and expression changes is key to classification accuracy. In this paper, an improved method for image feature extraction and representation on the basis of 2DPCA - fusion of 2DPCA and uniform rotation invariant LBP-was proposed. 2DPCA has many advantages over PCA. First, it is more straightforward to extract the facial image feature since it is based on two dimensional image matrixes. Second, the average recognition rate of 2DPCA is higher than PCA based on simulating experiments. As a method of texture analysis, LBP also has some unique advantages. The most superior properties of LBP are its tolerance of different illumination levels and its computational simplicity. Experimental results indicate that the combination of global features and local features is a simple but effective approach to facial image representation and face recognition.

\section{REFERENCES}

[1] A. Suruliandi, K. Meena, R. Reena Rose. 2012. Local binary pattern and its derivatives for face recognition. IET Com-puter Vision. 6(5):480-488.

[2] Drira, H. , Ben Amor, B. , Srivastava, A. 2013. 3D Face Recognition under Expressions, Occlusions, and Pose Variations[J]. IEEE Transactions on Pattern Analysis and Machine Intelligence, 35(9): 2270-2283.

[3] Guoying Zhao, Timo Ahonen, Jiř́ Matas. 2012. Rotation-invariant image and video description with local binary pat-tern features. IEEE Trans. on Image Processing. 21(4):1465-1477.

[4] Huang D, Shan C, Ardabilian M, Wang Y, Chen L. 2011. Local Binary Patterns and Its Application to Facial Image Analysis: A Survey. IEEE Trans on Systems, Man, and Cybernetics, 41(6):765-781.

[5] Kwak K, Pedrycz W. Face Recognition Using an En-hanced Independent Component Analysis Approach. Ieee T Neural Networ. 2007;18(2):530-541.

[6] Lee S, D Kim, J Cho. 2012. Illumination-robust Face Recognition System based on Differential Compo-nents[J]. IEEE Transactions on Consumer Electronics, 58(3): 963-970.

[7] Niraj P. Doshi, Gerald Schaefer. 2013. Texture classification using compact multi-dimensional local binary pattern de-scriptors.: IEEE, pp: 1-6.

[8] Ojala T, Pietikainen M, Maenpaa T. 2002. Multiresolution gray-scale and rotation invariant texture classification with local binary patterns. IEEE Trans. on Pattern Anal. 24(7):971-987. 
[9] Turk M, Pentland A. 1991. Eigenfaces for recognition. J Cogn Neurosci. [Journal Article]. 3(1):71-86.

[10] Yang J, Zhang D, Frangi AF, Yang JY. 2004. Two-dimensional PCA: a new approach to appearance-based face representation and recognition. IEEE Trans. on Pattern Anal Mach. 26(1):131-137.

[11] Wu Q, Wang M, Chai Z, Liang J. 2014. Different lighting processing and feature extraction methods for efficient face recognition. IET Image Processing. 8(9):528-538 